\title{
Implementation of an Improved Watershed Algorithm in a Virtex 5 Platform
}

\author{
Nadia Smaoui Zghal \\ Computers imaging and \\ Electronic Systems Group \\ ICOS research unit, ENIS, \\ Sfax, Tunisia
}

\author{
Khaled Taouil \\ UR CMERP National \\ Engineering School of Sfax, \\ Tunisia
}

\author{
Dorra Sellami Masmoudi \\ Computers imaging and Electronic \\ Systems Group ICOS research \\ unit, ENIS, \\ Sfax, Tunisia
}

\begin{abstract}
Watershed transform, as was described by Vincent and Soille, is a segmentation algorithm based on a flooding process of the gradient image, which is observed as a topographic surface. Watershed transform aims at finding the peaks in this surface and identifying them as image contours. This algorithm is used in many applications for its flexibility. However, its main limitation is the over segmentation. In this paper, we will try to overcome this limitation. The enhancement phase is based on filtering the original image by an anisotropic diffusion filter and then quantizing the gradient image. For evaluation, the improved algorithm is applied on an empirical basis for image segmentation research in order to be compared with other segmentation algorithms. Simulation results prove the effectiveness of our algorithm. Watershed algorithm is used in medical field in order to extract areas of interest in an image representing a section bone. Finally, the implementation phase in a Virtex 5 is based on the co design methodology. The synthesis results show that the performance of the developed design is $130 \mathrm{MHz}$ and the hardware occupation is about 78\% for an image of size of $256 * 256$.
\end{abstract}

\section{Keywords}

Watershed, quantization, anisotropic filter, Virtex 5, codesign.

\section{INTRODUCTION}

Image segmentation is an essential process for most image analysis tasks allowing partition an image into different regions and/or contours. Several techniques and algorithms have been developed for image segmentation such as clustering (exp: Kmeans)[2], edge detection algorithm (exp: Canny) [24] and region based methods. Watershed transformation is considered as a very useful and powerful tool for morphological image segmentation. It is used in many domains such as medical imaging [17] and especially for locating tumors [8], multi-spectral satellite imagery[18], machine vision, etc...With a moderate computational complexity, this algorithm is able to identify the important contours in a given image [3]. It is applied on a gradient image which is considered as a topographic surface and obtained by applying an appropriate morphological gradient operator on a grey scale image. Watershed algorithm consists in detecting the regional minima of the gradients and placing a water source in these minima in order to inundate the relief from sources. Then, it builds barriers when different sources are meeting. The resulting set of barriers constitutes the watershed.
In practice, this algorithm produces an important over segmentation due to local irregularities in the gradient image. A major improvement of the watershed algorithm consists in immersing the topographic surface with a previously defined set of markers [13]. Another approach for overcoming the over-segmentation problem is based on filtering the original image in order to anneal small peaks in the gradient which can be suspected as contours [11].

Here, in the improvement phase, we use an anisotropic diffusion filter applied to the original image aiming at smoothing the image by preserving the edges and then an inverse logarithmic quantification is applied to the gradient image in order to reduce the irregularities in this image.

The implementation phase of watershed algorithm in embedded system is considered as an important task. In this paper, we use the Software/Hardware co-design methodology for the implementation of the improved algorithm. In fact, using this methodology in such embedded system makes it possible to meet different design constraints such as size, cost and power dissipation.

This paper is organized as follows: section 2 presents our approach in watershed implementation for reducing over segmentation. The evaluation of our method compared to other algorithms and the simulation results are presented in section 3. In Section 4, we present the application of the improved watershed algorithm on an histological bone in order to be analyzed automatically. Section 5 deals with the implementation of the proposed architecture. Finally, we end with a brief conclusion.

\section{IMPROVINGWATERSHED BASED SEGMENTATION}

\subsection{Watershed basic algorithm}

In mathematical morphology, Watershed algorithm as was described by Vincent and Soille [14] is a segmentation algorithm based on the immersion principle. The algorithm is applied to a gradient image observed as a topographic surface. We flood this surface from its regional minima and we avoid the merging of the waters coming from different sources by constructing watersheds. Thus, we partition the image gradient into two different sets: the watershed lines and the catchment basins [8]. In this way, we obtain a segmentation of an image (figure 1). 


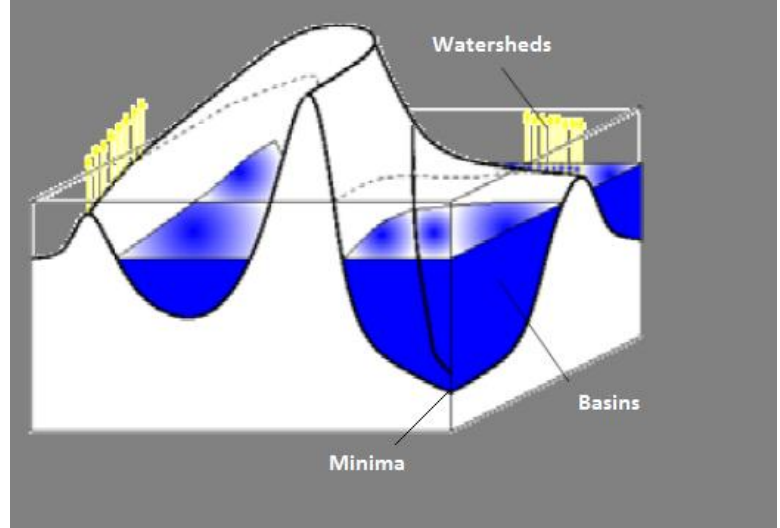

Fig1: Immersion principle

In fact, the algorithm operates in two stages: a sorting process followed by a flooding process. In the first step, the algorithm sorts the pixels in order of ascending grey scale values. During the flooding process, and for each grey level, the algorithm makes a test for each pixel. If the considered pixel has a labeled neighbor, it takes this label; otherwise it takes a new label. If it has two different neighbors, it is considered as belonging to watershed lines [15]. We have applied this basic watershed algorithm on a grey scale image; the result is presented in figure 2:

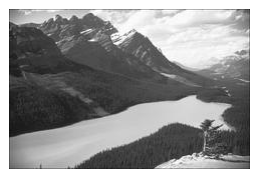

a)Original image

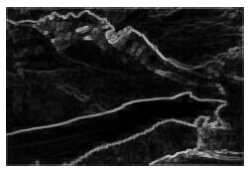

b)Gradient image

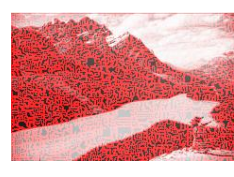

c)Segmented image

\section{Fig2: Application of a watershed algorithm on grey scale} Image

As can be shown, this method suffers from over segmentation. In fact, watershed transform considers in a similar way higher and lower valued gradients. In the following section, we explain how to resolve this limitation.

\subsection{Improving watershed algorithm}

\subsubsection{Anisotropic diffusion filter}

In the applications of object detection procedures, smoothing an image by preserving the edges is very important. The preprocessing filtering tool for edge detection and segmentation tasks should keep real contours in the image. In our context, anisotropic diffusion filter is used as a preprocessing tool to reduce bad gradient peaks in watershed. In fact, this filter is an iterative process. In each iteration, the image's gradient is calculated to detect the edges. For each pixel, a diffusion coefficient depending on the value of the gradient is computed. For low values of the gradient considered as homogenous zones of the image, the diffusion is allowed with a high diffusion coefficient. For the high values of the gradient considered as the edges, the diffusion is blocked by a weak diffusion coefficient [9]. Perona and Malik filter is based on the following equations:

$$
\frac{\partial \mathrm{I}(\mathrm{x}, \mathrm{y}, \mathrm{t})}{\partial \mathrm{t}}=\operatorname{div}(\mathrm{g}|| \nabla \mathrm{I}|| \nabla \mathrm{I})
$$

Perona and Malik suggested two different edge stopping functions $\mathrm{g}($.$) .$

$$
\begin{gathered}
g(\nabla \mathrm{I})=\frac{1}{1+\left[\frac{(|| \nabla \mathrm{I}||)}{\mathrm{k}}\right]^{2}} \\
\mathrm{~g}(\nabla \mathrm{I})=\mathrm{e}^{-}\left[\frac{(|| \nabla \mathrm{I}||)}{\mathrm{k}}\right]^{2}
\end{gathered}
$$

The stopping function $\mathrm{g}$ contains the parameter $\mathrm{K}$. This parameter corresponds to the height of contours to preserve. The anisotropic diffusion filter decreases the over segmentation resulted from the application of the watershed. In this paper, we use equation 3 and the choice of the parameters is purely experimental; the number of filter's iterations is equal to 30 and $\mathrm{k}$ is equal to 5 .

The application of the watershed algorithm on the grey scale image which is filtered by an anisotropic filter is presented in the figure 3 .

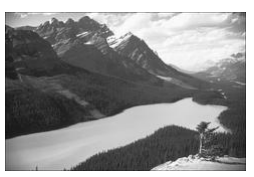

a)Original image

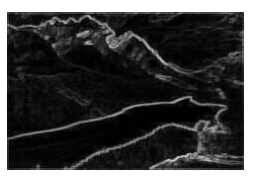

b)Gradient image

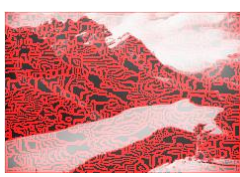

c)Segmented image

\section{Fig3: Segmentation result for an image filtered by an anisotropic filter}

Despite the application of the anisotropic filter permits to ameliorate the segmented image, the over segmentation persists. So, to solve this problem, we should minimize the insignificant basins. That's why we had the idea of quantizing the gradient image.

\subsubsection{Inverse logarithmic quantization of the gradient image}

The over segmentation problem is raised when watershed basic algorithm creates a basin for each local minimum. For that, we had the idea to reduce the basins by minimizing insignificant local minima. To do so, it's necessary to quantify the gradient image in such a way that we preserve the high intensity levels and we reduce the low intensity levels. In fact, an object contour correspond generally to high levels of gradient in the image, contrariwise, local minima, leading to over segmentation problem, belong generally to object surfaces or backgrounds and corresponds accordingly to low intensity levels in the gradient image.

On another side, the logarithmic quantization quantifies the pixels in areas of low intensity with relatively small steps and quantifies the pixels in areas of high intensity with large steps. Thus, we can exploit this idea by using the intervals found by the logarithmic quantization in the inverse direction. This research is based on the Weber's law [10] which reflects a logarithmic perception of the eye to the light. The Weber's law is expressed as follows:

$$
\frac{\Delta \mathrm{I}}{\mathrm{I}}=\mathrm{K}
$$

Where $\Delta \mathrm{I}$ represents the difference threshold, I represents the initial intensity and $\mathrm{k}$ which is the Weber fraction means that the proportion $\Delta \mathrm{I} / \mathrm{I}$ remains constant despite variations in the I term. 
We established the Weber's equation for different values of $\mathrm{k}$. The experimental study permits to find good segmentation results for $\mathrm{k}=0.1$. The following figure (Fig.4) presents the dictionary values.

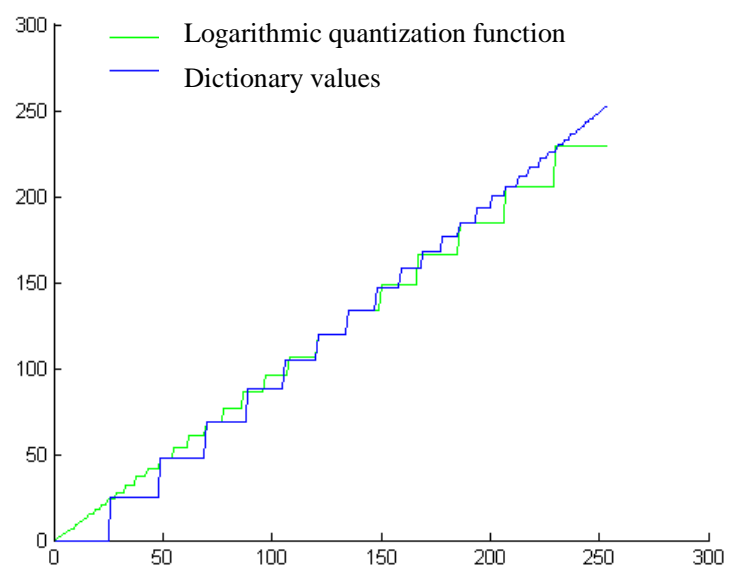

Fig4: Dictionary Values

\section{EVALUATION AND COMPARISONS}

In the literature, many criteria for the evaluation of image segmentation are proposed. These criteria can be divided into two main categories: supervised evaluation and unsupervised evaluation. In the latter case, we can evaluate the quality of the image segmentation without having a reference image. But in the case of the supervised evaluation, the segmented image is evaluated when compared to a known reference called ground truth, created manually by experts [5]. Figure 5 illustrates the principle of the supervised evaluation.

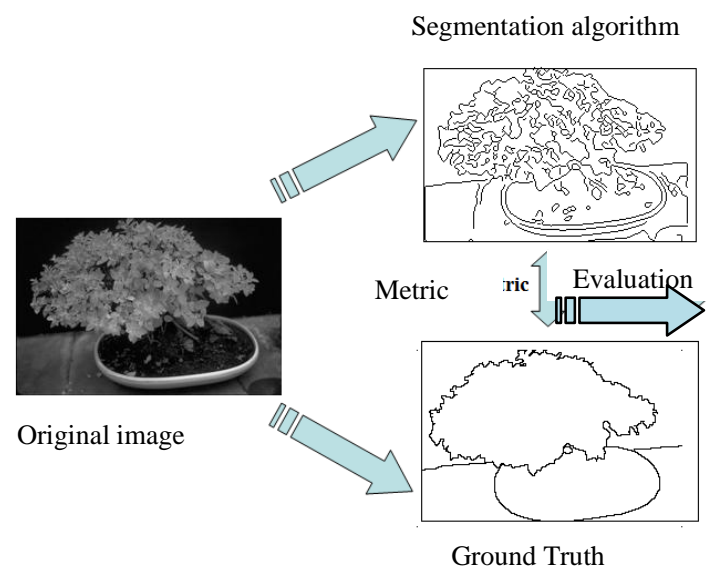

Fig5: Supervised evaluation procedure of a segmentation result

In this paper, we use some images selected from the Berkley database. This database is an empirical basis for image segmentation research [21]. Figure 6 shows five images selected from each category in this database and the corresponding ground truth. The evaluation of the segmentation algorithms is based on three error measures:

- Under detection error corresponds to pixels of Iref which have not been detected

$$
U D E=\frac{\operatorname{card}\left(I_{R e f} / I_{F}\right)}{\operatorname{card}\left(I_{\text {Ref }}\right)}
$$

- Over detection error corresponds to detected contours which do not coincide with Iref

$O D E=\frac{\operatorname{card}\left(I_{F} / I_{R e f}\right)}{\operatorname{card}(I)-\operatorname{card}\left(I_{R e f}\right)}$

- Localization error computes the distance between the misclassified pixels and the nearest pixels of Iref

$$
L E=\frac{\operatorname{card}\left(I_{R e f} / I_{F} \cup I_{F} / I_{R e f}\right)}{\operatorname{card}(I)}
$$

With Iref is the reference contour which corresponds to the ground truth and IF is the detected contour obtained through the segmentation result of an image I. A segmentation result is considered good for small values of the three errors [19].

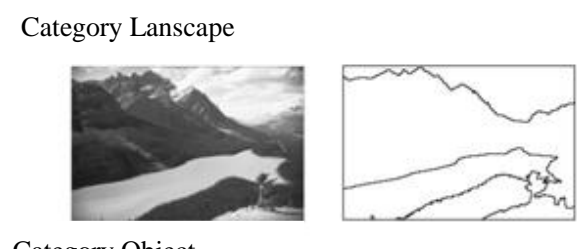

Category Object
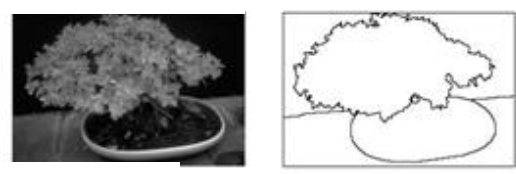

Category Animal
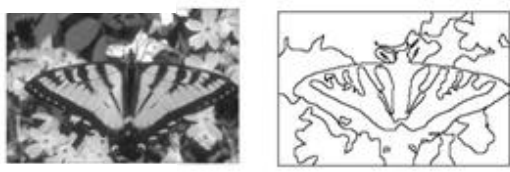

Category Nature
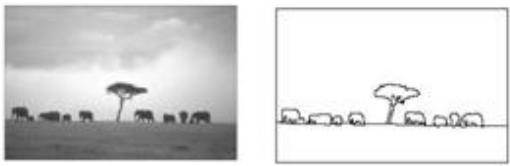

Category People
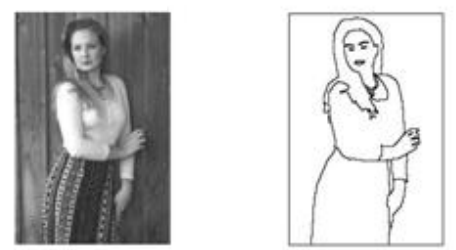

Fig6: Some images selected from the Berkley database and the proposed contours

\subsection{Comparison with the basic Watershed algorithm}

In the first step, we evaluate our improved algorithm by comparing it to the basic one (Watershed algorithm with no enhancement). Figure 7 shows the application of the two methods on 5 images selected from each category in the 
Berkley database. The table 1 presents the error measures obtained for 100 images selected from this database.

This table indicates that our algorithm is well improved compared to the basic one. In fact, for the three error measures, values are considerably lowered (values in bold indicate the best segmentation result).

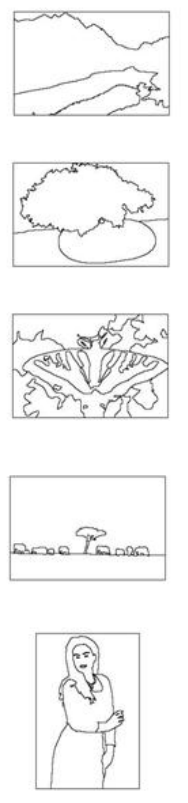

a)
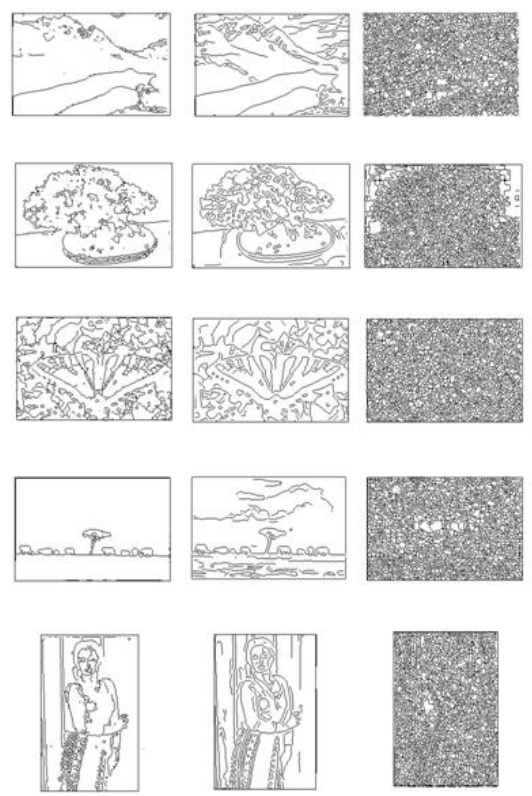

b)

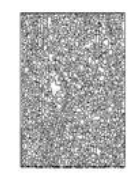

d)
Fig7: Image segmentation: a) Manual contours as proposed in the Berkley database, b) Contours obtained

by our improved watershed algorithm, c)Contours obtained by Canny operator, d) Contours obtained by the basic watershed

Table 1. Performance measures obtained from the watershed basic algorithm and our improved algorithm

\begin{tabular}{|l|c|c|c|}
\hline Category & $\begin{array}{c}\text { Error } \\
\text { Measure }\end{array}$ & $\begin{array}{c}\text { Basic } \\
\text { Watershed }\end{array}$ & $\begin{array}{c}\text { Our } \\
\text { algorithm }\end{array}$ \\
\hline \multirow{4}{*}{ Landscape } & Over & 0.9486 & 0.8165 \\
\cline { 2 - 4 } & Under & 0.0516 & 0.0279 \\
\cline { 2 - 4 } & Loc & 0.4768 & 0.06425 \\
\hline \multirow{3}{*}{ Object } & Over & 0.9599 & 0.8109 \\
\cline { 2 - 4 } & Under & 0.0540 & 0.0200 \\
\cline { 2 - 4 } & Loc & 0.2122 & 0.06315 \\
\hline \multirow{3}{*}{ Nature } & Over & 0.9256 & 0.8269 \\
\cline { 2 - 4 } & Under & 0.0945 & 0.0566 \\
\cline { 2 - 4 } & Loc & 0.2907 & 0.1350 \\
\cline { 2 - 4 } & Over & 0.9530 & 0.7275 \\
\cline { 2 - 4 } & Under & 0.0461 & 0.0274 \\
\hline \multirow{3}{*}{ People } & Loc & 0.1321 & 0.0502 \\
\cline { 2 - 4 } & Over & 0.9511 & 0.8892 \\
\cline { 2 - 4 } & Under & 0.0634 & 0.0375 \\
\cline { 2 - 4 } & Loc & 0.2396 & 0.1446 \\
\hline
\end{tabular}

\begin{tabular}{|l|c|c|c|}
\hline \multirow{3}{*}{ Average } & Over & 0.9476 & $\mathbf{0 . 8 1 4 2}$ \\
\cline { 2 - 4 } & Under & 0.0619 & $\mathbf{0 . 0 3 3 8}$ \\
\cline { 2 - 4 } & Loc & 0.2703 & $\mathbf{0 . 0 9 1 4}$ \\
\hline
\end{tabular}

\subsection{Comparison with the canny operator}

The second step is based on comparing the improved algorithm to canny operator [24]. To do so, we apply this operator, considered as an efficient segmentation algorithm, on the same images selected from the Berkley database (figure 7). Table 2 presents the error measures obtained respectively by canny operator and the enhancement algorithm. In this table, values in bold indicate the best segmentation result.

Starting from table 2, we note that for the over-detection and the localization errors, values obtained by our improved algorithm are lower than that obtained by canny operator. For the under detection error, better result is obtained by canny operator. But it is very proximate to that obtained by our algorithm. Thus, our enhancement algorithm is close to if not better than canny operator which proves the effectiveness of our method.

Table 2. Comparison between the improved algorithm and canny operator

\begin{tabular}{|l|c|c|c|}
\hline Category & $\begin{array}{c}\text { Error } \\
\text { Measure }\end{array}$ & $\begin{array}{c}\text { Canny } \\
\text { Operator }\end{array}$ & $\begin{array}{c}\text { Our } \\
\text { algorithm }\end{array}$ \\
\hline \multirow{4}{*}{ Landscape } & Over & 0.8919 & 0.8165 \\
\cline { 2 - 4 } & Under & 0.0279 & 0.0279 \\
\cline { 2 - 4 } & Loc & 0.1043 & 0.06425 \\
\hline \multirow{4}{*}{ Object } & Over & 0.8995 & 0.8109 \\
\cline { 2 - 4 } & Under & 0.0207 & 0.0200 \\
\cline { 2 - 4 } & Loc & 0.0955 & 0.06315 \\
\hline \multirow{3}{*}{ Nature } & Over & 0.8163 & 0.8269 \\
\cline { 2 - 4 } & Under & 0.0573 & 0.0566 \\
\cline { 2 - 4 } & Loc & 0.1220 & 0.1350 \\
\cline { 2 - 4 } & Over & 0.8096 & 0.7275 \\
\cline { 2 - 4 } & Under & 0.0253 & 0.0274 \\
\hline \multirow{3}{*}{ People } & Loc & 0.0784 & 0.0502 \\
\cline { 2 - 4 } & Over & 0.8984 & 0.8892 \\
\cline { 2 - 4 } & Under & 0.0316 & 0.0375 \\
\cline { 2 - 4 } & Loc & 0.1177 & 0.1446 \\
\hline \multirow{3}{*}{ Average } & Over & 0.8631 & $\mathbf{0 . 8 1 4 2}$ \\
\cline { 2 - 4 } & Under & $\mathbf{0 . 0 3 2 5}$ & 0.0338 \\
\cline { 2 - 4 } & Loc & 0.1036 & $\mathbf{0 . 0 9 1 4}$ \\
\hline
\end{tabular}

\section{IMPLEMENTATION OF THE PROPOSED ARCHITECTURE IN A VIRTEX 5 PLATFORM}

In this paper, we are based on the co-design methodology for the implementation of the whole system described in section 2 on a Virtex 5 platform: the Xilinx XC5VFX70T. This latter, belongs to Xilinx Virtex 5 development platform series. The heart of this board is a Virtex 5 FPGA chip which has a PowerPC Processor surrounded by FPGA fabric [22]. This architecture offers a big flexibility by the method of hardware 
and software co-design. The software application is running on the PowerPC processor, while FPGA is used for hardware acceleration [7]. The aim of this work is reducing design time and reaching better performances when implementing the developed architecture with hardware and software resources. Thus, for design optimization, we use Co-developer of Impulse Accelerated technologies, which is a C-to-HDL compiler. This tool permits to realize all the stages from initial design to final implementation of the considered algorithm [20]. In the first stage which is the simulation, the source code of the design is described in C-language and then simulated based on Impulse C. In the second stage which is the partitioning, the source code is divided into hardware and software components. For this co-design project, software part consists of the input and output interfacing.

The hardware part operates on the greyscale image stored in the memory in order to perform the image processing task. Therefore, the compiler is used for generating automatically the appropriate interfaces for each part. Finally, a configuration bit stream is generated based on the Studio Design Kit from Xilinx used to configure the FPGA on the Virtex 5 platform [22]. We notice that for an image of the size of $256 * 256$ the system runs at $130 \mathrm{MHz}$ and occupies $78 \%$ of the hardware resources.

\section{CONCLUSION}

In this paper, we present an improved watershed algorithm. The enhancement of this algorithm is based on filtering the original image by an anisotropic diffusion filter and then quantifying the gradient image based on an inverse logarithmic quantization. For evaluation, the proposed architecture is applied on an empirical database for research on image segmentation and boundary detection in order to be compared with other segmentation algorithms. Simulation results prove the performance of our algorithm. Moreover, this paper is based on the co-design methodology for the implementation of the improved architecture in a Xilinx Virtex 5 development platform. The software component which is the interfacing of the input and output image is running on the PowerPC processor. The hardware part, which is the anisotropic diffusion filter, the gradient operator and the watershed algorithm co-process with the PowerPC to achieve the goals of acceleration. . This strategy leads to acceptable hardware resources occupation (about 78\%) and to a frequency performance of approximately $130 \mathrm{MHz}$ for an image of size of $256 * 256$.

\section{ACKNOWLEDGEMENTS}

We sincerely thank:

- Laboratoire d'histologieembryologie, Faculté de Médecine de Sfax

- Unité Sciences et Technologies de l'Image et des Télécommunications de l'Institut de Biotechnologie de Sfax

\section{REFERENCES}

[1] A.Michael Parfitt, Mark k.Dreznzr, Francis H.Glorieux, Jhon A.Kanis, Hartmut Malluche, Pierre J.Menuier, Susan Mott and Robert R.Recker «A bone histomorphometry: Standardization of Nomenclature. Sumbols and Units», Journal of bone and Mineral Research, Volume 2, Number 6, 1987.

[2] Bo Zhao, Zhongxiang Zhun, Enrong Mao and Zhenghe Song «Image Segmentation Based on Ant Colony
Optimization and K-Means Clustering» College of Engineering China Agricultural University Beijing. China, 2007.

[3] C. Rambabu. I. Chakrabarti and A. Mahanta «Floodingbased watershed algorithm and its prototype hardware architecture» IEE Proc.-Vis. Image Signal Process, Volume. 151. Number. 3, June 2004.

[4] Carolina A. Moreira Kulak, David W. Dempster «Bone histomorphometry: a concise review for endocrinologists and clinicians», Arq Bras Endocrinol Metab, 2010.

[5] DO Minh Chau «Evaluation de la segmentation d'images », Institut de la francophonie pour l'informatique, Vitnam, 2007.

[6] Eric Lespessailles, Christine Chappard, Nicolas Bonnet, Claude Laurent Benhamou, «Imaging techniques for evaluating bone microarchitecture». Elsevier, Revue du Rhumatisme 73 (2006) 435443.

[7] G. F. Zaki. R. A. Girgis. W. W. Moussa , and W.R. Gobran. Junior Member. IEEE «Using HW/SW Codesign to Implement an Embedded Face Recognition/ Verification System on an FPGA».

[8] H.S.Sheshadri and A. Kandaswamy «Detection of Breast Cancer Tumor based on Morphological Watershed Algorithm» Department of ECE. PSG College of Technology. Coimbatore.

[9] Hye Suk Kim, Hyo Sun Yoon, Nguyen Dinh Toan and Guee Sang Lee «Anisotropic Diffusion Transform based on Directions of Edges» Korea IEEE 8th International Conference on Computer and Information Technology Workshops.

[10] H.LOUKIL HADJ KACEM and M.S BOUHLEL «Elaboration d'une Quantification Semilogarithmique pour l'Amlioration de la Norme de Compression JPEG», 5th International Conference: Sciences of Electronic. Technologies of Information and Telecommunications March 2009, TUNISIA.

[11] K.Karantzalos. D. Argialas «Improving edge detection and watershed segmentation with anisotropic diffusion and morphological levellings» Remote Sensing Laboratory, School of Rural and Surveying Engineering (SRSE), National Technical University of Athens (NTUA), Athens. Greece June 2006.

[12] Wafa Abid. Khaled Taouil. Tarek Rebai , Med Salim Bouhlel «Segmentation d'image par seuillage d'histogramme application l'analyse des coupes histologiques osseuses». 3rd International Conference: Sciences of Electronic. Technologies of Information and Telecommunications, Tunisia, 2005.

[13] Li Gao, Shuyuan Yang, Jie Xia, Junli Liang . Yuhua, Qin «A new marker-based watershed algorithm», China 2006

[14] Luc Vincent and Pierre Soille "Watersheds in Digital Spaces: An Efficient Algorithm Based on Immersion Simulations " $0162 \quad$ i $8828=91=0600$; 058301:0001991IEEE 
[15] N. Smaoui Zghal. A. Yangui Jammoussi. D. Sellami Masmoudi «Implementation of a watershed based image segmentation system in Virtex II pro platform» DTIS 2009. Cairo. Egypt

[16] M.-H. Lafage-Proust «Peut-on optimiser l'evaluation du statut osseux chez l'insuffisant renal par les explorations complementaires ?». Flammarion Mdecine- SciencesActualits nephrologiques 2009.

[17] P. Dillinger. J. F. Vogelbruch. J. Leinen. S. Suslov. R.Patzak. H. Winkler. and K. Schwan «FPGA-Based Real-Time Image segmentation for Medical Systems and Data Processing» IEEE Transactions on nuclear Science VOL. 53. NO. 4. August 2006

[18] S.Chen1. J.Luo1. Z.Shen1. X.Hu1. L. Gao1 "Segmentation of Multi-spectral Satellite Images Based on Watershed Algorithm" Institute of Remote Sensing Application. Chinese Academy of Scienses. Beijing. 100101. China 2008

[19] S.Chabrier. H.Laurent. C.Rosenberger, "Supervised evaluation of synthetic and real contour segmentation results".14 th European Signal Processing Conference. Italy.2006

[20] Accelerate Software Algorithms on FPGAs, available at www.impulseaccelerated.com/eval/index

[21] Computer Vision Group, available at www.eecs.berkeley.edu/Research/Projects/CS/vision /grouping/segbench/

[22] Xcell Journal, available at www.Xilinx.com

[23] Xiutao Shi . X. Sherry Liu . XiangWang . X. Edward Guo. Glen L. Niebur « Effects of trabecular type and orientation on microdamage susceptibility in trabecular bone».Elsevier. Bone 46 (2010)

[24] Xun Wang and Jian-Qiu Jin «An Edge Detection Algorithm Based on Improved CANNY Operator» College of Computer and Information Engineering Zhejiang Gongshang University. Hangzhou. 310035 China 0-7695-2976-3/07 5:002007 IEEE. 